Revue des patrimoines

$40 \mid 2019$

Lits historiques. Première anthologie des lits européens du XVe au XIXe siècle

\title{
Betten fränkischer Fürsten im Spätmittelalter
}

\section{Uta Piereth}

\section{(2) OpenEdition}

Journals

Édition électronique

URL : http://journals.openedition.org/insitu/23604

DOI : 10.4000/insitu.23604

ISSN : 1630-7305

Éditeur

Ministère de la Culture

Référence électronique

Uta Piereth, «Betten fränkischer Fürsten im Spätmittelalter », In Situ [En ligne], 40 | 2019, mis en ligne le 18 septembre 2019, consulté le 04 octobre 2019. URL : http://journals.openedition.org/insitu/23604 ; DOI : 10.4000/insitu.23604

Ce document a été généré automatiquement le 4 octobre 2019.

\section{(c) $(1)$}

In Situ Revues des patrimoines est mis à disposition selon les termes de la licence Creative Commons Attribution - Pas d'Utilisation Commerciale - Pas de Modification 4.0 International. 


\title{
Betten fränkischer Fürsten im Spätmittelalter
}

\author{
Uta Piereth
}

\section{Zollern in Franken - Ausgangspunkt und Rahmen der Überlegungen}

Die hier präsentierten Recherchen standen in Zusammenhang mit einer konkreten Aufgabe: Die Bayerische Verwaltung der Staatlichen Schlösser, Seen und Gärten plante für 2017 die Eröffnung eines großen Burgmuseums in der ursprünglich überaus bedeutenden und stattlichen Cadolzburg nahe Nürnberg, dem fränkischen Herrschaftsmittelpunkt der Hohenzollern im späten Mittelalter (Abb. 1). 
Abb. 1

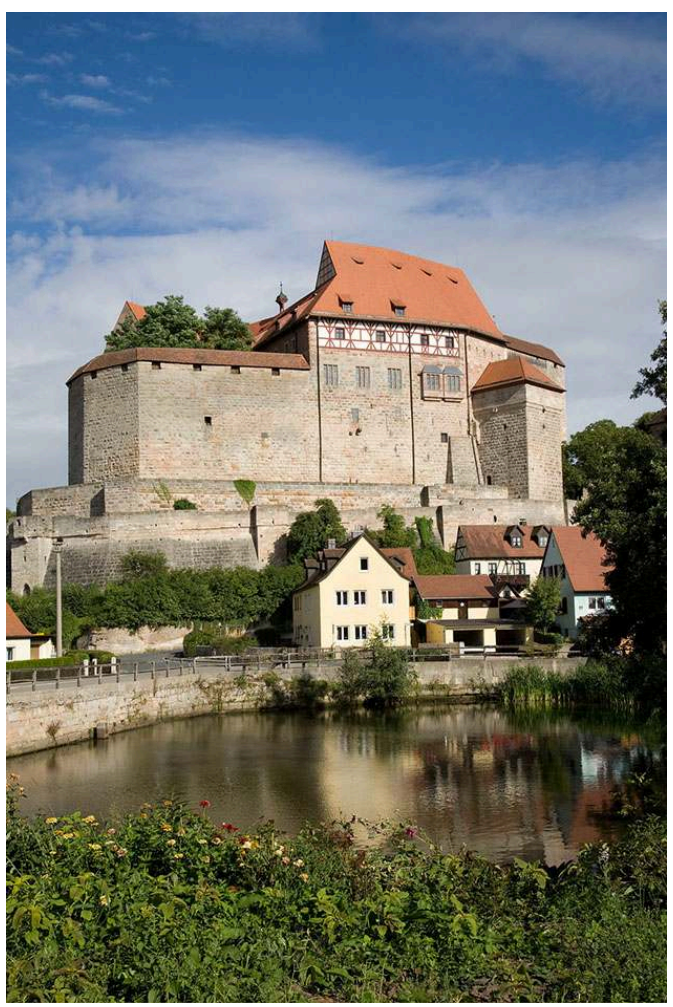

Cadolzburg von Westen.

Foto: Andrea Gruber. (c) Bayerische Schlösserverwaltung, 2013

2 Die Dynastie der damals nur «Zollern» genannten Herren wurzelte in Schwaben. Doch gelang ihnen ab etwa 1200 von Franken aus eine wahrhaft steile Karriere an der Seite der deutschen Könige, zunächst als Burggrafen von Nürnberg, sodann als Fürsten und ab 1415/17 schließlich als Kurfürsten der Mark Brandenburg. Sie waren damit einer der sieben Königswähler im Reich, mithin eine der mächtigsten Familien der Zeit ${ }^{1}$. Die Zollern regierten fortan also ebenso über Brandenburg im Norden und Franken im Süden des Reichs. Dabei galt es, zahlreiche bewaffnete Konflikte zu bewältigen, eine enorme Schuldenlast und die wenig effiziente Verwaltung der Mark zu parieren. Erst ab etwa 1470 steuerte Kurfürst Albrecht Achilles (1414-1486) seine zweigeteilte Herrschaft im Spagat von Franken aus durch konsolidiertere Zeiten, ungeachtet seines militärischen Engagements auch in Reichskriegen wie jenen gegen Karl den Kühnen 1474 am Rhein. Dieser (Kur-)Fürst war sich seines Ranges sehr bewusst. Er legte Wert darauf, dies anlässlich von Festen und Jagdausflügen, aber auch mit einer standesgemäßen Lebenshaltung in Cadolzburg, Ansbach, Kulmbach oder Berlin-Cölln zum Ausdruck zu bringen, wie wir aus zahlreichen Dokumenten wissen².

Angesichts der Bedeutung der Dynastie, des Ortes und der in Cadolzburg herrschenden Persönlichkeiten ist es besonders bedauerlich, dass sich für die Einrichtung eines Museums dort kaum Originalobjekte erhalten haben, unter anderem da der Bau selbst am Ende des Zweiten Weltkriegs massive Zerstörungen erlitten hatte und ab 1979 baulich erst wieder gesichert und zugänglich gemacht werden musste ${ }^{3}$. Die Motivation jedoch, just hier die politische und Alltagsgeschichte der Zollern im 15. Jahrhundert museal nachvollziehbar zu machen, veranlasste das Team der Schlösserverwaltung dazu, möglichst viel zu visualisieren und erlebbar werden zu lassen, was man an Hand 
der unterschiedlichsten Quellen auch über die Raumdisposition der Burg, ihre Nutzung und die Lebensformen darin an Informationen ziehen kann.

In diesem Zusammenhang stellte sich auch die Frage, was über das Thema Kammer am Hof dieser Herren zu sagen wäre. Was ließe sich tatsächlich herausfinden und museal verständlich machen zu der Überlegung, wie der Fürst, seine Frau und der Hofstaat schliefen? Zusammen mit Tanja Kohwagner-Nikolai suchten wir hierzu einschlägiges Material. Glücklicherweise ist es um schriftliche Quellen rund um die Hohenzollern der Epoche besser bestellt als um die Objektüberlieferung, diese sind daher zuerst zu erörtern.

\section{Schriftliche Quellen}

\section{Zeitgenössische Quellen}

5 Für die Zollern und Franken im 15. Jahrhundert verfügen wir vor allem über zwei Inventare. Das eine stammt aus dem Jahr 1471. Albrecht Achilles veranlasste, dass die Basisbestände der Güter in seinen fränkischen Herrschaftssitzen erfasst werden in einem Moment, als er selbst mit seinem eigentlichen Hofstaat für geraume Zeit nach Brandenburg aufbrach. Was dort zu Ansbach notiert ist, dem Schloss, das zunehmend die Funktion einer Residenz übernehmen sollte, gibt bereits Aufschluss über die Nomenklatur und über die vorgesehenen bzw. vorhandenen Bettstätten. Dabei sei vorab in Erinnerung gerufen, dass in Deutschland solche (Schlaf-)Kammern in der Regel ungeheizt waren ${ }^{4}$. An mobilem Inventar sind hier unter anderem für die Ehefrau des Kurfürsten zwei Fürstenbetten, drei kleine Betten, eine rote Decke, zwei Herrenund ein zwei kurze Polster verzeichnet ${ }^{5}$. In der Kammer der Hofdamen standen dem Inventar zufolge sechs Betten mit Polstern, Leintüchern und diverse Decken, in derjenigen des Kurfürsten selbst ein Fürstenbett samt -Polster, ein Deckbett und ein feines Paar Leintücher sowie zwei weitere Bettstätten ${ }^{6}$. Außerdem sind in der Silberkammer Stoffe und Bettwäsche verzeichnet: einfache oder feine Leintücher, seidene Bettdecken, Kissen aus Samt und Seide sowie, separat aufbewahrt in einer eigenen Truhe, Leintücher mit goldenen Borten oder Kissen mit gewirkten Bordüren ${ }^{7}$. Hier finden sich also Anhaltspunkte für die Anzahl der fest vorgesehenen Betten des an sich noch immer ambulanten Hofes sowie die fixe Mindestausstattung an verwendeten Stoffen und Bettauflagen, die später noch genauer zu interpretieren sind. Zwar wird auch im Jahr 1544 nochmals ein Inventar erstellt, doch ist zu diesem Zeitpunkt Cadolzburg nur noch eine gelegentlich genutzte Nebenresidenz, für die 23 Matratzen, 24 Decken, 16 «Pfülben» und 18 Kissen vorgehalten wurden ${ }^{8}$. Für die Plassenburg, die damals gerade zu einer Festung umgerüstet wurde, beschreibt ein weiteres Verzeichnis 1532 die dortige Bettausstattung mit immerhin unter anderem 147 Federbetten, großteils mit Bezügen aus schwerem Zwillich für die Dienerschaft, sechs Unterbetten in Fürstenqualität, 98 Kissen in Zwillich- oder Leinenbezügen und sechs Fürstenkissen. Außerdem hielt man 58 Polster vor, davon 12 in guter Qualität ${ }^{9}$. Wenn also der Markgraf hier mit kleinem Gefolge vorbeikam, war man präpariert.

Das zweite Inventar, das für unsere Zeit und Verhältnisse zur Orientierung dienen kann, stammt ebenfalls aus dem Jahr 1471, wo man in Nürnberg den Besuch von Kaiser Friedrich III. auf der Kaiserburg erwartete. Der Baumeister Endres Tucher erfasste detailliert, was für diesen Anlass vorgesehen war. Auch wenn es die kaiserliche, nicht 
eine (kur-)fürstliche Unterbringung beschreibt, hat man so doch einen guten Anhaltspunkt für die Ausstattung einer hochrangigen Kammer, sofern sie einen reisenden Herrscher beherbergte. So ist die Rede von einem zu diesem Zweck aufgeschlagenen großen «spanpet» mit Strohsack und zwei Federbetten darauf, mit goldenen Kissen und Polster, einer roten Seidendecke («golter») und einem Vorhang ${ }^{10}$. Es handelte sich in diesem Fall also um ein Gurt- oder Spannbett - im Unterschied zu den bei den Hohenzollern erwähnten «Herrenbetten», die wohl eher solche mit Bretterböden bezeichneten. Auch ein Baldachin war vorgesehen, von dem allerdings nicht klar ist, ob es sich um einen halben oder vollen Himmel handelte handelte; immerhin scheint es zum fraglichen Zeitpunkt ein übliches Attribut gehobener Bettausstattung gewesen zu sein. Auch Kissen und Decken in ähnlich edlen Stoffen dürften für eine fürstliche Bettausstattung ebenfalls anzunehmen gewesen sein ${ }^{11}$.

7 Schaut man sich speziell im Ambiente der wohlhabenden Reichsstadt Nürnberg, die in der Region und darüber hinaus durchaus Maßstäbe setzte, näher an, so kann man zum einen auch eine Quelle eher literarischen Charakters mit einbeziehen. Der Nürnberger Dichter und Meistersinger Hans Folz (1440-1513) beschreibt in seinem Buch über den «Hausrat» die Kammer Nürnberger Bürger, die mit einem «süßen und weichen» (Gurtoder Spann-) Bett samt Strohsack und Matratze, Decke, diversen Kissen, Polstern und Leinen ausgestattet war $^{12}$. Auf der Basis zahlreicher schriftlicher Quellen erforschte Zander-Seide ${ }^{13}$ die konkreten Materialien, die beim Nürnberger Patriziat im 15. und 16. Jahrhundert für ihre Betten Verwendung fanden. Ihre Befunde bestätigten das im oben zitierten Inventar der Zollernfürsten benutzte Vokabular. Zander-Seidel übersetzte dieses im materiellen Sinn, was sehr hilfreich ist bei der Einschätzung der in der fränkischen Oberschicht verbreiteten Stoffe und Qualitätsniveaus. Sie präzisierte, welche Stoffe hier verfügbar waren oder gar in Nürnberg selbst produziert wurden: Typischerweise waren es Zwilch, Barchent, der ab 1535 in der Stadt hergestellt wurde, außerdem Stücke, die unter der Bezeichnung «Nürnberger Decken» bis nach Italien und Skandinavien Verbreitung fanden, sowie «Golter» als abgenähte Bettdecken. Bettdecken aus Leder, Atlas aus Brügge oder Nürnberg (ab 1530), aus Damast, Taft, «flidt seiden» oder in «türkischer» Art konnte Zander-Seidel ausmachen sowie die Art, wie Kopfkissenbezüge verschlossen wurden.

\section{Frühneuzeitliche Inventare}

8 Zur Abrundung des archivalischen Befundes konsultierten wir auch Kammer-Inventare anderer Epochen und Schlösser in Bayern. Zunächst sind die Cadolzburger Inventare von 1699 und 1789 zu erwähnen. Damals wurde die Cadolzburg als markgräfliche Nebenresidenz genutzt und verfügte über aktuell umgebaute Gebäudeteile mit fürstlichen Appartements. Es waren darin 1699 an Bettzeug Dutzende Oberbetten aus Barchent, Zwillich und Federn, Pfülben aus Zwillich und Federn, Kopfkissen aus Barchent, teils weiß-blau bezogen, und Federn und sonstige Polster und Kissen genannt ${ }^{14}$. 1789 schließlich ging die Beschreibung auch auf die Farbigkeit der Textilien im Schlafzimmer Markgräfin ein, wo viel grüner Brokat, grüne Fransen, grüner Seidenatlas samt Borten, Himmel und mit grünem Tuch bedecktem Fußboden nebst etlichen Möbeln genannt wurde ${ }^{15}$ : Erst zu diesem späten Zeitpunkt sind also andere und differenziert bezeichnete Ausstattungselemente und Stoffe belegt. Wirft man einen Blick in andere hochwertige Schlossausstattungen, etwa die der Grafen von Ortenburg 1696 oder in der Residenz der Kurfürsten von Bayern in München $1769^{16}$, so finden sich 
in beiden Fällen noch mehr oder weniger die gleichen Elemente wie jene des für das späte Mittelalter benannten: Strohsäcke, Unterbetten oder «madrazen» aus Barchent (inzwischen mit Rosshaarfüllung), «Polster» oder Keile analog zu den «pfülben», verschiedene Decken aus Taft, Baumwolle, Atlas oder Damast, (nun auch mit Federfüllung), mit Goldborten oder einfarbig, im Fall des Gesindes kariert oder gestreift; die Stoffe der Himmel waren in den gleichen Farben ausgewiesen wie die Deckbetten (couvertures doublées). Auch die Art, wie die verschiedenen Unterbetten aufeinander lagerten und wie man sich bettete mit Keilen und mehreren Kissen, ähnelt sich über die Jahrhunderte hinweg. Es gibt also tatsächlich eine Art Sach- oder Liegeüberlieferung, auch wenn natürlich der Komfort und die Erlesenheit mancher Stoffe noch zugenommen haben mögen.

\section{Bildquellen}

9 Die dritte Quellenart ist bildlicher Natur: Gemälde, Graphiken und andere Bildwerke, bei denen das Bettmotiv vorkommt. Im späten Mittelalter taucht es in Franken vor allem in sakralen Tafelgemälden auf. Mit einer Szene aus dem Marienleben sei beispielsweise ein Werk von Sebastian Deyg in Heilsbronn, dem Begräbniskloster der Zollern, aus dem Jahr 1511 erwähnt. (Abb. 2)

Abb. 2

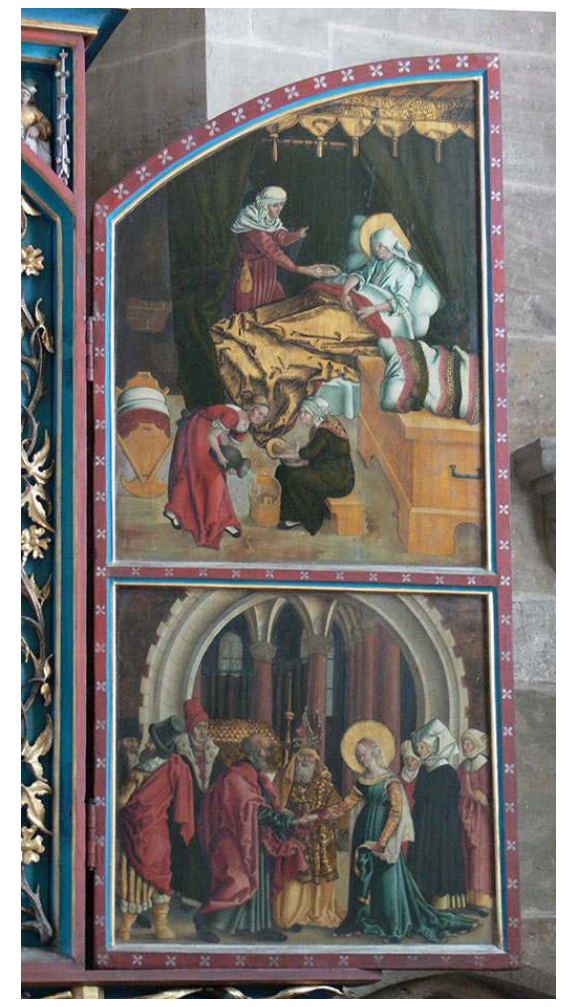

Sebastian Dayg: Geburtsszene, Detail aus Marienaltar Heilsbronn, 1511.

Foto: Albert Dietl. (C) Albert Dietl, 2014.

Maria liegt hier in einem Bett unter grünem Baldachin, der mit goldgelben Fransen und Borten verziert ist, mit verschiedenen, ebenfalls teils golden anmutenden Decken, feinen Leintüchern und gestreiftem Kissen. Andere Werke aus dem Umfeld Albrecht 
Dürers zeigen ähnliche Dispositionen ${ }^{17}$. Von Dürer selbst sind Holzschnitte aufschlussreich wie jener des Hl. Hieronymus (1492) oder vom Marientod 1510, bei dem unter anderem eine interessante Art ersichtlich ist, den Himmel des - im übrigen nicht mit Stoff bekleideten - Baldachins an der Decke zu befestigen. (Abb. 3)

Abb. 3

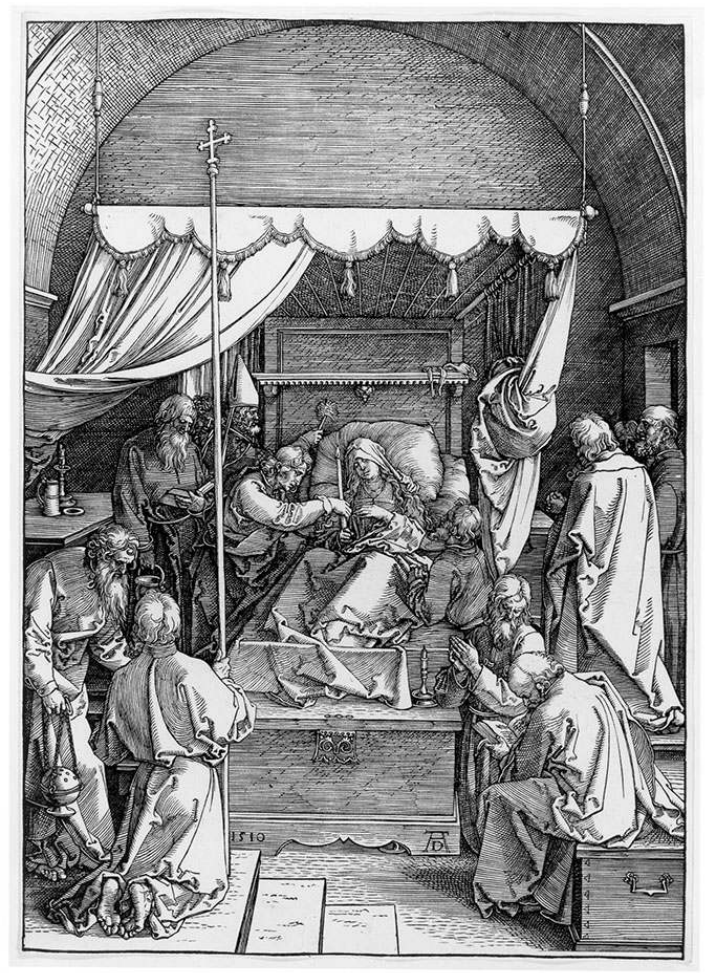

Albrecht Dürer: Marientod, 1510. Staatliche Graphische Sammlung München (1920:148 D).

(c) Munich, Staatliche Graphische Sammlung (1920:148 D)

Betrachtet man über Franken hinaus Bildbeispiele aus Süddeutschland allgemein, gibt es verschiedene Werke, die weitere Auskunft über die verbreitete Art der Bettkonstruktionen und -ausstattung erteilen. Es finden sich darin gerne hölzerne skulptierte, aber nicht unbedingt textil bekleidete Kopfteile oder Himmel (teils mit demi-ciel courbé), die Vorhänge sind oft schlicht und grün, die Decken ebenfalls grün und hochwertig in der Anmutung ${ }^{18}$.

\section{Erhaltene Betten}

Kammerausstattungen aus Franken, speziell von den Zollernfürsten haben sich aus dem späten Mittelalter nicht erhalten - bedauerlicherweise auch nicht jene Bettstatt des Albrecht Achilles, von dem noch im 19. Jahrhundert ein Historiker berichtet: einem großen Bett aus Zedernholz und Elfenbein, das (erst) im Jahr 1802 verkauft worden sei $^{19}$. Dagegen existieren noch zwei sehr aufwendige Betten aus etwas jüngerer Zeit (um 1600). Eines steht in Kulmbach, wo die Plassenburg eine der wichtigsten Residenzen der Hohenzollern war. Sobald 1603 die brandenburgische Linie der Hohenzollern das Erbe ihrer fränkischen Verwandten angetreten hatten, verlegte zwar Markgraf Christian von Brandenburg-Bayreuth mit seiner Gemahlin Marie seine Hauptresidenz nach 
Bayreuth, doch ließen die beiden die Festung Plassenburg ihrem Rang gemäß neu ausstatten. Dazu gehörte auch das außergewöhnliche Bett (Abb. 4), das über einem gelängten achteckigen Grundriss mit acht Säulen einen Baldachin mit Glashimmel hat. Das ganze Bett aus Eichenholz ist ornamental in Gold und Braun über grünem Grund gefasst. Ursprünglich sollen die Vorhänge mit Perlen besetzt gewesen sein, doch verkaufte man in späteren Zeiten die Perlen ${ }^{20}$. Skulpturen der Caritas und Pietas (aus Lindenholz) flankieren heute noch die Kopfbretter, ein Amor überflog in der Originalausstattung das Lager. Das ikonographische Programm passt demnach perfekt zum Funktion eines fürstlichen Brautbettes.

Abb. 4

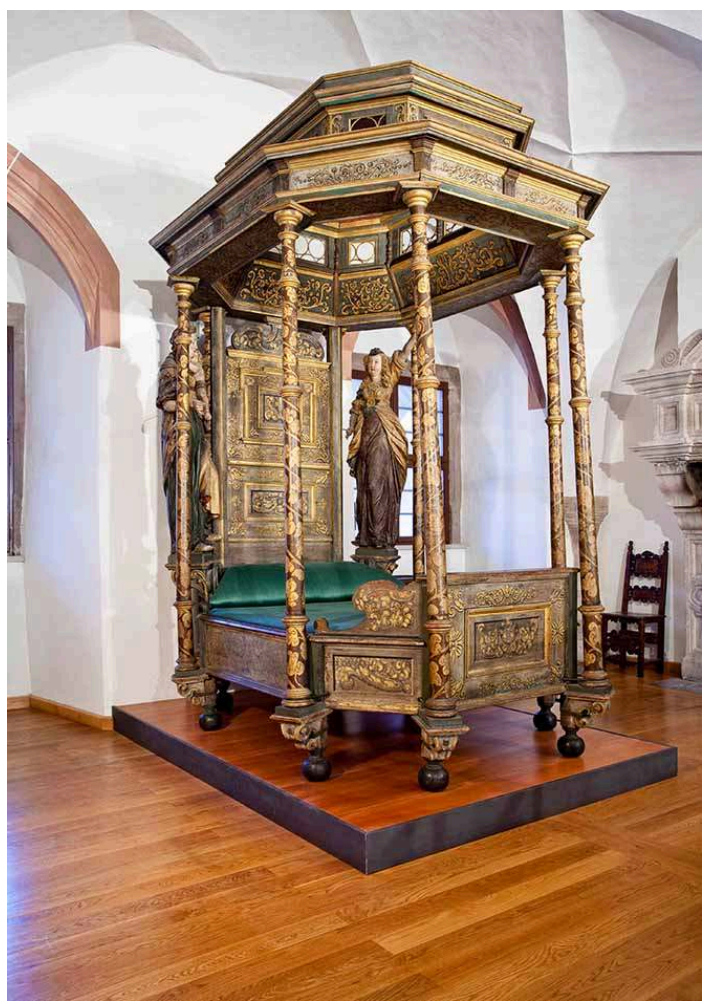

Bett in der Plassenburg, 1604. Dauerleihgabe des Bayerischen Nationalmuseums München.

Foto: Thomas Köhler. @ B Bayerische Schlösserverwaltung.

Ein zweites Bett der Hohenzollern, diesmal aus der Ansbacher Residenz, steht heute im Bayerischen Nationalmuseum (Abb.5). Es handelt sich um ein Reisebett Markgraf Georg Friedrichs (gest. 1603). Die Ausarbeitung ist äußerst hochwertig mit Marketerien aus Ebenholz, Elfenbein, Obst- und Tropenhölzern, mit einem Baldachin und ursprünglich Seidenvorhängen. Damit ist das hohe Ausstattungsniveau belegt selbst für Zeiten, als die fränkischen Hohenzollern nicht mehr dieselbe eminente Bedeutung hatten wie im 15. Jahrhundert. 


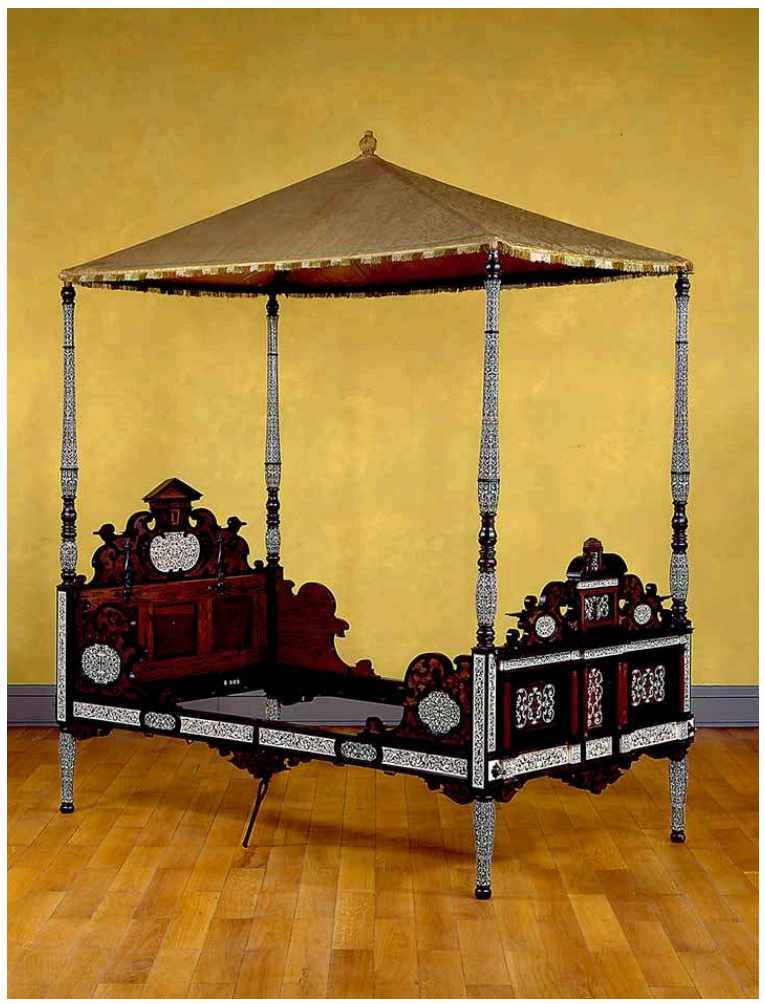

Reisebett aus Ansbach in der Art Peter Flötners, Augsburg, um 1600. Bayerisches Nationalmuseum München (R 909).

Foto: Walter Haberland. (c) Münich, Musée national bavarois (R 909).

Ebenfalls im Bayerischen Nationalmuseum haben sich Bettgestelle aus dem Spätmittelalter erhalten, wenngleich nicht aus dem Kontext des Zollernhofes. Aus der Alpenregion etwa wird dort ein Bett des 15. Jahrhunderts aufbewahrt aus Zirbenholz (pin montagnard) mit skulptiertem Kopfteil. Von einem anderen Exemplar, einem Kastenbett, erhielten sich fein durchbrochene Maßwerkschnitzereien vom Originalkopfteil ${ }^{21}$.

Was die textile Ausstattung angeht, so gibt es in den Depots des Germanischen Nationalmusems in Nürnberg zwei besonders interessante Stücke ${ }^{22}$ : ein Kopfkissenbezug aus feinstem Leinen vom späten 16. Jahrhundert, durch den die Art, den Bezug mit Schlaufen zu verschließen, belegt ist, sowie eine Bettdecke von einem Mann, der in Nürnberg großen Einfluss hatte, mithin aus der Nachbarschaft und Einflusszone der Hohenzollernresidenzen. Diese große Decke weist Seidenstickerei über schwarzem Wollgrund auf. Wappen, Inschriften und Blumen zieren sie. Die Machart, der Reichtum und die Ikonographie ebenso wie ihre Maße geben Aufschluss über die dazugehörige Bettstatt und deren hochwertige stoffliche Ausstattung ${ }^{23}$. Mir scheint gewiss, dass wir uns die Bettdecken der Hohenzollernfürsten eher noch wertvoller vorzustellen haben.

\section{Die Kammer in der Cadolzburg}

16 Trotz der Zerstörung des Alten Schlosses in der Cadolzburg durch den Brand 1945 haben wir eine recht präzise Vorstellung vom historischen Vorzustand. Dies ist vor 
allem sehr exakten Bauaufnahme-Zeichnungen des Burgenforschers Bodo Ebhardt $\mathrm{zu}$ verdanken, der 1905/1906 viele Monate dort verbrachte. Ergänzend existieren einige Schwarz-Weiß-Fotografien des Vorkriegszustandes. Im zweiten Obergeschoss lag das kurfürstliche Appartement, es war vor allem im seinerzeit als «Stube» bezeichneten Hauptraum sehr repräsentativ ausgestattet von den Dimensionen und den architektonischen Bauformen mit Schmuckkamin, sechs Erkern und komplexen Sterngewölben her. Im Geschoss darüber lagen hochwertig mit geschnitzten Vertäfelungen an Decken und Wänden ausgestattete Räume, die so das «Frauenzimmer» beherbergt haben dürften (Abb. 6). Im 15. Jahrhundert war der Hof auch im räumlichen Sinn - etlichen Veränderungen unterworfen aufgrund der stets wachsenden Familie, die adäquat hier unterzubringen war. Einer der Räume jener Zeit dürfte ziemlich sicher als (nicht geheizte) Kammer der Gemahlin von Albrecht Achilles, der Kurfürstin Anna (1437-1512) gedient haben.

Abb. 6

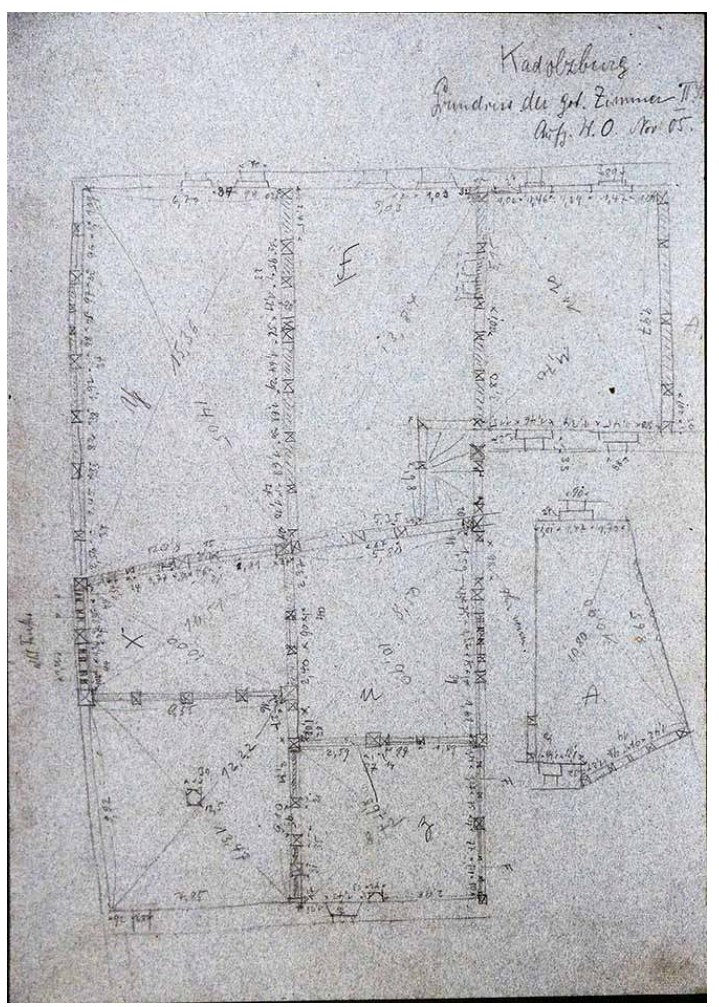

Bodo Ebhardt: „Grundriss der gotischen Zimmer“ im 3. Obergeschoss der Cadolzburg, („z" bezeichnet den Ort, der heute im Museum als Kammer eingerichtet ist), 1905. Europäisches Burgeninstitut -

Einrichtung der Deutschen Burgenvereinigung e.V. (Braubach): DBV-Archiv, Plansammlung Kadolzburg (K_001_144)

(c) DBV-Archiv, Plansammlung Kadolzburg (K_001_144).

Um den Besuchern der Cadolzburg zumindest eine Vorstellungshilfe der damaligen Situation vermitteln zu können, wurde der entsprechende Ausstellungsraum ähnlich dimensioniert und aus der aktuellen Heizschleife teilweise herausgenommen, so dass es in der Heizperiode spürbar kühler ist als im restlichen Museum. Ein Teil der Decke präsentiert sich anknüpfend an die dokumentierten Vorkriegsabbildungen mit geschnitzten Ornamenten und in verschiedenen, möglichen Farbfassungen ${ }^{24}$. Vor allem aber zieht eine fürstliche Bettstatt die Aufmerksamkeit der Besucher auf sich, in die 
man sich hineinlegen kann, um den Liegekomfort auf fünf verschiedenen Matratzenlagen, Keilen und Kissen auszuprobieren (Abb. 7) ${ }^{25}$.

Abb. 7

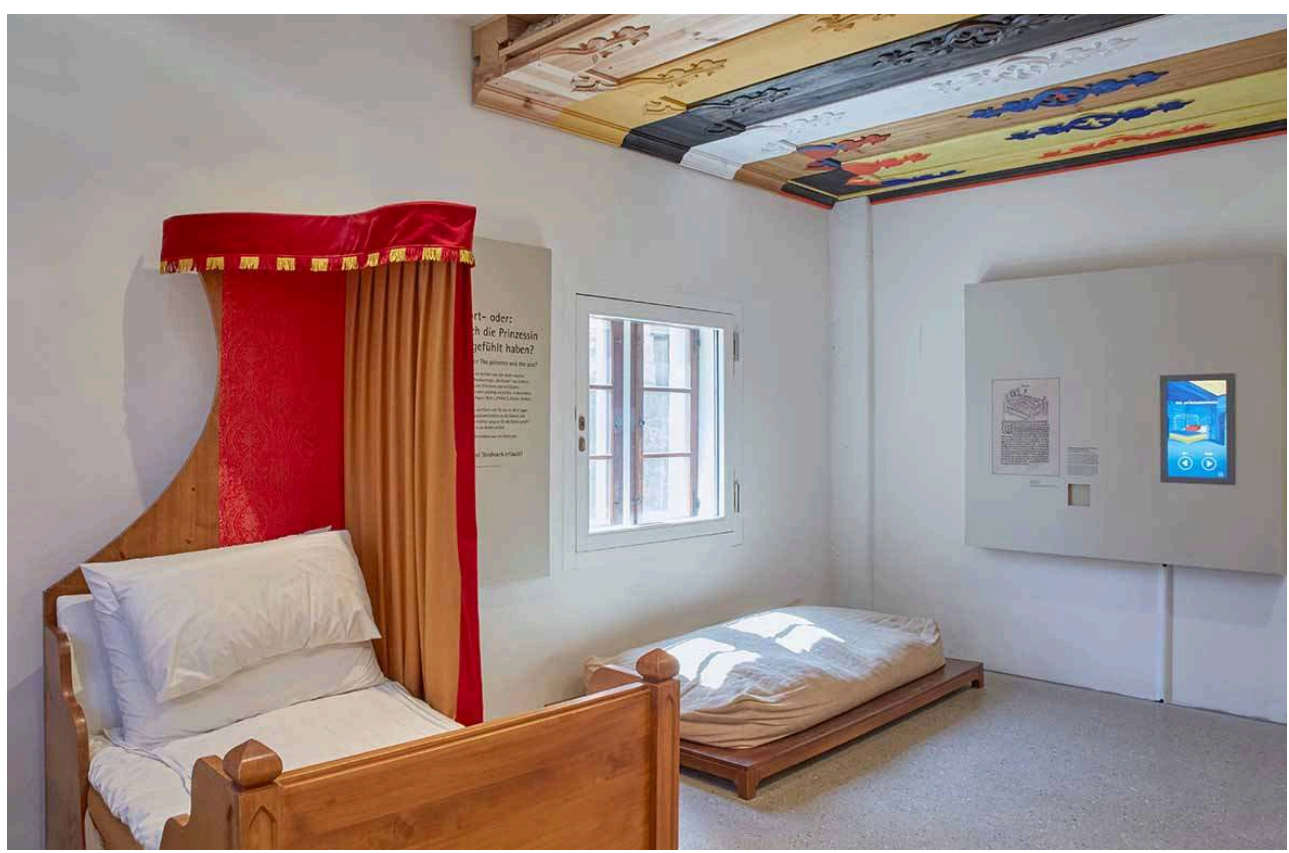

Fürstliche Kammer in der Cadolzburg, museale Präsentation Cadolzburg 2017.

Foto Bayerische Schlösserverwaltung, Maria Scherf/Andrea Gruber, 2018.

Die Basis der materiellen Umsetzung war das, was hauptsächlich aus dem Inventar von 1471 und einigen anderen Quellen an Informationen abzuleiten war. Daher soll kurz dargelegt werden, welche Schlüsselbegriffe insbesondere der höfischen BettenWeichteile dort auftauchen und wie wir sie interpretierten ${ }^{26}$. Im Inventar ist von mehreren «Herrenbetten» die Rede: Wir nehmen an, es handelte sich um Betten mit Baldachin auf Spanngurt- oder Bretterböden ${ }^{27}$. Die Bettauflagen wurden in diesem Inventar nicht näher beschrieben, doch weiß $\operatorname{man}^{28}$, dass es wohl auch Eichenblättermatratzen oder solche aus Adlerfedern in Lederhüllen für «Herrenbetten» oder Wollauflagen, in Hüllen ebenfalls aus behandelter Wolle, gab. «Lange Herrenpolster», «kurze Polster», «Polster» oder auch «pfülben» und «hauptstuck» waren verteilt auf die Menge an Betten: Normalerweise verfügte jedes Bett über mindestens ein «Polster», im Fall des Herrenbettes auch über mindestens zwei, die wohl mit Federn oder Wolle gefüllt waren. «Kws» oder «kuslein» meinten Kissen in verschiedenen Größen. In der Silberkammer wurden auch Kissen in Rot und Grün, Seide und Samt aufbewahrt ${ }^{29}$. «Kulter» oder «kotzen, golter» meinte eine Bettdecke, durchaus auch aus Seide und sehr hochwertig, eventuell bestickt, manchmal gesteppt und mit Daunen oder Schwanenfedern gefüllt ${ }^{30}$. «Deckbetten/Decken» bezeichnete Decken, die man während der Abwesenheit des Fürsten getrennt aufbewahrte. Es gab gemusterte oder farbige, solche aus Wolle oder anderen Qualitäten, gefüttert mit «zwilch» oder Seide. «Leilach» hießen die Leintücher, die im Inventar zu Dutzenden und immer paarweise aufgeführt wurden, unterschieden in «heren leilach», also feines Leinen, und "gemaine leilach» aus ungebleichtem Leinen oder Barchent. Der Begriff 
«Schallawn» tauchte ebenfalls auf und bezog sich auf die Herkunft eines Stoffes aus feiner, robuster Wolle.

Wegen des hohen finanziellen und konservatorisch-präventiven Aufwandes, den eine textile Annäherung an ein spätmittelalterliches Fürstenbett bedeutet hätte und eine interaktive Nutzung unmöglich erscheinen ließ, entschieden wir uns für eine reine Annäherung an das heute ungewöhnliche Liegegefühl in angewinkelter Haltung (bei 15-20'-Winkel), auf vielen Unterlagen und Stützhilfen. Auch Baldachin und Vorhänge aus Brokatstoff und Wollfutter wurden nur angedeutet, nicht rekonstruiert. Für den dauerhaften musealen Einsatz wählten wir moderne, robuste Füllungen und Überzüge an Stelle der organischen wie Adler- und Daunenfedern, Laub und feinstem Leinen. Um die ursprünglich verwendeten Materialien wenigstens zu zeigen, werden sie in zwei Röhren in Schichtmodellen präsentiert: für die Bettauflagen eines Herrenbettes und daneben im Vergleich eines Höflings (Abb. 8). Aus der Perspektive musealer Vermittlung mittelalterlicher Gegebenheiten erwies sich diese Herangehensweise seit der Eröffnung der Cadolzburg als ein sehr geglücktes Element.

Abb. 8

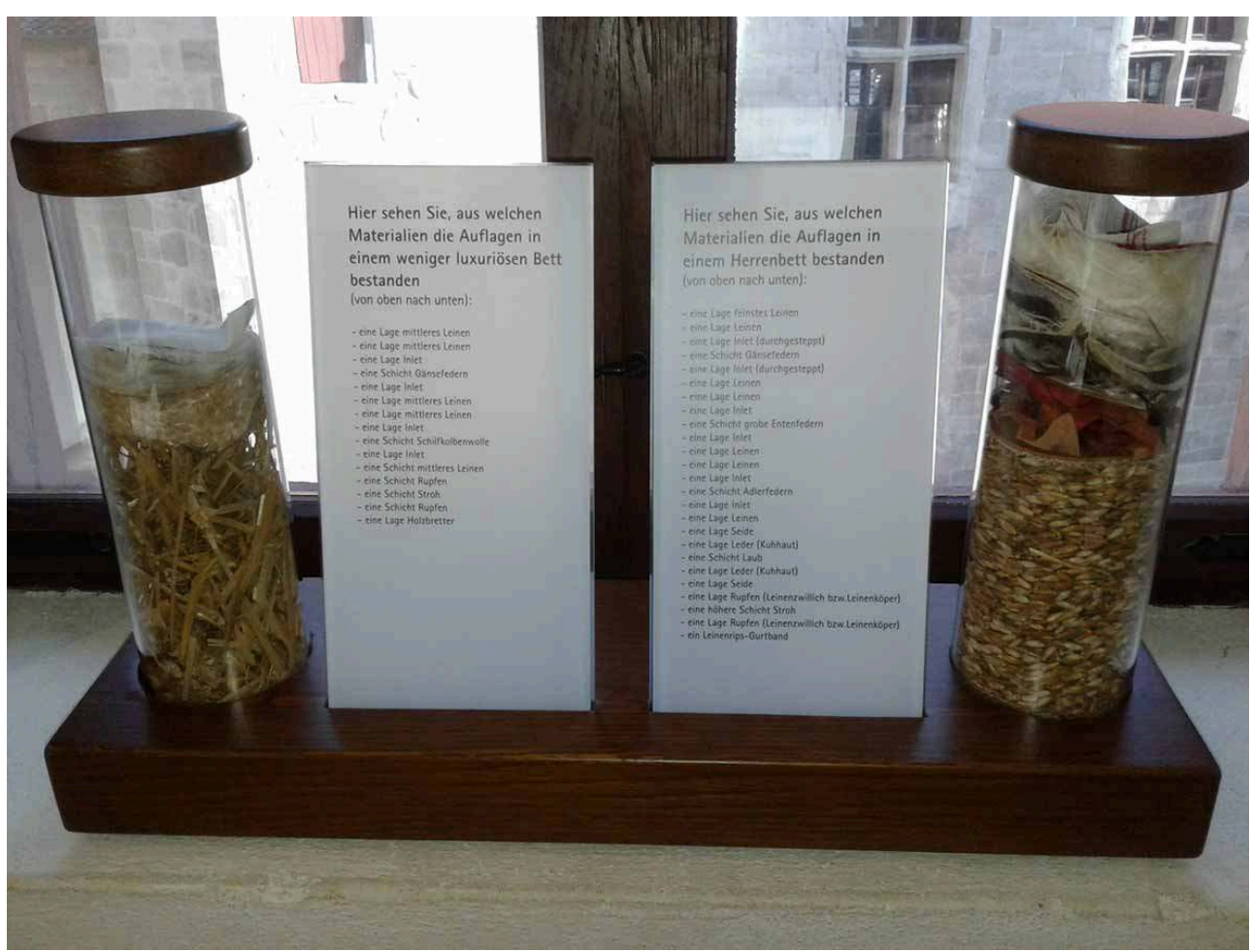

Museale Darstellung jener Materialien, wie sie für die Ausstattung spätmittelalterlicher Betten am Fürstenhof benutzt worden sein dürften. Kammer in Cadolzburg.

Foto: Uta Piereth, 2017. (c) Uta Piereth. 


\section{NOTES}

1. - s. KARNATZ, Sebastian/PIERETH, Uta (Hg.): «herr im hauß». Die Cadolzburg als Herrschaftssitz der fränkischen Zollern im Mittelalter. München 2017, darin bes. SEYBOTH, Reinhard: «Die Karriere der Hohenzollern im späten Mittelalter: Ein Spagat zwischen Franken und Brandenburg, Land und Reich», S. 10-27, zur kaiserlichen Anerkennung fürstengleichen Ranges 1363 und der Belehnung mit der Mark Brandenburg faktisch 1415 und formal auf dem Konstanzer Konzil 1417 S. 15-17. Zur Cadolzburg als Museum s. www.burg-cadolzburg.de.

2. - s. PIERETH, Uta: «Herrschaftszeiten: Der Zollernhof und die Cadolzburg unter Friedrich I. und Albrecht Achilles.» In: «herr im hauß». Die Cadolzburg als Herrschaftssitz der fränkischen Zollern im Mittelalter. KARNATZ Sebastian/PIERETH, Uta (Hg.). München 2017, S. 28-72. Außerdem MÜLLER, Mario (Hg.): Kurfürst Albrecht Achilles (1414-1486). Kurfürst von Brandenburg. Burggraf von Nürnberg. Ansbach 2014. NOLTE, Cordula: Familie, Hof und Herrschaft. Das verwandtschaftliche Beziehungs- und Kommunikationsnetz der Reichsfürsten am Beispiel der Markgrafen von Brandenburg-Ansbach (1440-1530). (Mittelalter-Forschungen 11), Ostfildern 2005.

3. - s. BURGER, Daniel: «Die Cadolzburg 1486 bis 1945 - Bau und Funktion». In: «herr im hauß». Die Cadolzburg als Herrschaftssitz der fränkischen Zollern im Mittelalter. KARNATZ Sebastian/PIERETH, Uta (Hg.). München 2017, hier S. 153-159, sowie BAUER, Jürgen: «Der Wiederaufbau der Cadolzburg - zwischen Bewahrung des Baudokuments und aktuellen Nutzungsanforderungen», ebda. S. $160-173$.

4. - s. HOPPE, Stephan: Die funktionale und räumliche Struktur des frühen Schloßbaus in Mitteldeutschland. Untersucht an Beispielen landesherrlicher Bauten der Zeit zwischen 1470 und 1570. Köln 1996, bes. S. 366f.

5. - Codex diplomaticus Brandenburgensis. Sammlung der Urkunden, Chroniken und sonstigen Quellenschriften für die Geschichte der Mark Brandenburg und ihrer Regenten. RIEDEL, Adolph Friedrich (Hg). Band III, 2, Berlin 1860, S. 47-50 am 4. Oktober 1471, hier S. 47: «In meiner gnedigen frawen Camern vnd Stuben: II heren peth, I rothe deck, III cleine peth, II lang heren polster, II kurtz polster.»

6. - Codex/RIEDEL III, 2 ebda., S. 47f.: «In des Jungen heren knaben Cameren: III pethlein, III pose leilach, II polster, I polsterlein, III schallawn. » «In der Jungen frewlein Cameren» mit einigen Hofdamen, einem Narr und zwei kleinen fürstlichen Kindern befanden sich neun Bettstätten, darunter für den Prinzen ein wohl kleineres «pethlein» mit «kuslein» und «deckpethlein»; der Narr Hamlein hatte bei seiner Bettstatt eine Decke, Leinwand und ein ledernes Polster. «In meins gnedigen heren Cameren: I heren peth, I heren polster, I deck peth, I par heren leilach. Im andern peth: I peth, I polster, I par leilach, hat der kugler, ist I alt (I news), I deck Peth, I rote deck. Im dritten peth: I peth, I polster, I deckpeth.»

7. - Codex/RIEDEL III,2 ebda. S. 49 zur Silberkammer, wo u.a. aufgeführt sind: «I heren par leilach, I par gemaine leilach, I deckpetlein meiner gnedigen fraw», auch Zwillich mit Borten, «I par heren leilach, I Seiden golter mit I ploen vnterzug, I kus, III tewich, II alte samete kws, sind rot, I Seiden kus grun, I alter peth zichen» sowie in einer Extratruhe, zusammen mit sakralen Gegenständen: «1 par leilach mit gulden leisten, 1 gestraimpten strosack, 6 grose heren kws, 1 kleins kus,kus mit einer gewurckten zichen, 3 par gemaine leilach. ».

8. - Staatsarchiv Nürnberg Fm Ansbach Rep. 103a II Brand. Lit. 2305, hier u.a.: «Vnderbeth klein Vnnd groß» und 3 «Bar furstenbeilager».

9. - WEIGAND-KARG, Sabine: Die Plassenburg. Residenzfunktion und Hofleben bis 1604. Weißenstadt 1998, S. 344.

10. - LEXER, Matthias/WEECH, Friedrich von (Hg.): Endres Tuchers Baumeisterbuch der Stadt Nürnberg (1464-1475). Stuttgart 1862, S. 299f: «item in des keisers grün kamer des keisers groß 
spanpet, 1 strohsack, 2 federpet ob einander, 4 leilach dorauf gepreit, von golcz 1 polster, 2 küss uberzogen mit golcz und tolden von weissen garen an den zipfelen, doruber gedeckt ein roten seiden golter; umb das pet was ein furhang von ploben scheter gemacht, und 2 nider penck auf den peden seitten desselben spanpecz. Item in derselben kameren waren 2 spanpet, in iedem ein strosack, 1 federpet, 1 polster, 2 küss, 2 leilach, 1 deck; mer dorinnen 1 lange taffel auf 2 pocken und 2 fürpenck vor den peten; die glesser vor denselben fensteren in derselben kameren furgeseczt. »

11. - Weitere Anhaltspunkte verschafft ein Rechnungsbuch, das im Jahr 1500 Anschaffungen für die Kaiserburg verzeichnet: Michel Behaim kaufte z.B. ein «seyden votter golter» für 14 Gulden (!), ein anderes und zwei «seyde genet deck» sowie zahlreiche Federbetten, Decken und Leintücher, s. Staatsarchiv Nürnberg Rep. 2 c 227 Heft No. 1.

12. - Gedichte vom Hausrat aus dem 15. und 16. Jahrhundert. Hg. HAMPE, Theodor. Straßburg 1899, darin Hans Folz, S. 4: «In die schlaffkamer do ich schleich/der hat ein pet seüst unde weich/rüet die nacht des seuberleicht/irt die nit was/dicz oder das/so schlafft ir also frey - / Ein stro sack spanpet und ein deck/ein deckpet wü es nit will kleck/Schaw das in nit der winter weck/küs polster leylach mit/nacht schüch nacht haüben zimet aüch/wer dar on spart der ist ein gaüch/ die mon zw notürfft dut gepraüch/ein prüncz scherben/drüen kisten/ die sach hat gar kein pit...»- Die deutsche höfische Literatur beschreibt Betten eher im semiotischen Sinn. Häufig ist hier das Bett ein quasi-öffentlicher Ort oder aber schlicht Zeichen des edlen Standes des Protagonisten, was auch an den beschriebenen Materialien (Seide, Gold etc.) ablesbar wird, zum Beispiel beim „Eneas“ des Heinrich von Veldekke oder dem Straßburger «Alexander»; vgl. dazu LERCHNER, Julia: Lectulus floridus: zur Bedeutung des Bettes in Literatur und Handschriftenillustration des Mittelalters. Köln 1993.

13. - ZANDER-SEIDEL, Jutta: Textiler Hausrat. Kleidung und Textilien in Nürnberg 1500-1650. München 1990, besonders S. 334-370.

14. - BURGER, Daniel: Die Cadolzburg. Dynastenburg der Hohenzollern und markgräflicher Amtssitz. (Wissenschaftliche Beibände zum Anzeiger des Germanischen Nationalmuseums Bd. 24. Wartburg-Gesellschaft zur Erforschung von Burgen und Schlössern e.V. Forschungen zu Burgen und Schlössern Sonderband 1). Nürnberg 2005, S. 182: (1699) «An bettgewandt: Zwey oberbett mit barcheten ingefüder. Zwey oberbett mit claren zwillich ingefüder. Zwey pfülben mit zwiller. Ein unterbett mit federrieth. Zwey unterbett mit federrieth. Vier oberbett mit barchet. Zwey oberbett mit federriethen. Drey pfülben von zwillich. Drey pfülben von federrieth. Zehen kopffküßen von barchet. Zwey kopffküßen von federrieth. Ein deckbett von zwillich. Fünff deckbett von barchet. Acht unterbett von zwillich. Zehen kopffküßen von barchet. Fünff deckbett. Fünff unterbett. Fünff polster. Sieben küßen. Zwey oberbett von federrieth. Zwey unterbett von dergleichen, Zwey unterbett von zwillich. Zwey polster von zwillich. Zwey polster von zwillich. Sieben küßen. Ferner an bettwerck: Zwölff oberbett, darunter drey weiß und eine blau bezogen. Sechzehen unterbett, alle blau bezogen. Sechs und zwanzig pfülben, darunter 22 blau bezogen. Zwanzig und neün kopfküßen ohnbezogen.» nach StAN Rep.225/4 I Rentamt Cadolzburg 193, Bausachen, Bd. 2.

15. - BURGER, Daniel, ebda. S. 182 (1789) «1 tapeten von grünem procatel. 2 tisch nebst denen teppichen von grünen mogat, das hochfürstliche bett auswendig mit einem procatel samt denen 2 bonne grace mit grün seidenen kreppenfranzen ausstaffirt, inwendig von grün seidenen atlas mit seinen borten besetzt, nemlich der himmel, das küpfstück, die obere decken, benebst denen inwendigen falbeln, und das bett aussen herum, der vorhand von grünen sasfiant, der fußboden, worauf das bett stehet, mit grünen tuch bedeckt, welch letzteres aber verfault und abg. ist. 1 spiegel mit einem rahm von braunem holz. 2 wandleuchter gleich den obigen. 2 armlehnssessel. 6 chaisen und 4 tabouretts mit grünen mogat bezogen. 1 weis leinwandener vorhang mit 6 blättern, und mit grünen franzen eingefaßt. 1 kleiner caminschirm mit vergoldetem holzrahm und inwendig eingemachten figuren.» nach StAN Rep. 225/4 RA Cad. Nr. 780. 
16. - Bayerisches Hauptstaatsarchiv (BHStA) Ortenburg-Archiv Akten 0 2417: Inventarium über die mobilien und wahrnus bey dem Hochgräfl. Schloß Alten Orttenburg Anno 1696 S. 4-7, 9f., 15-17. Inventar der Residenz München s. Bayerische Schlösserverwaltung, Museumsabteilung, Inventar 1769.

17. - Z.B. Michael Wolgemut: Szenen aus dem Marienleben, Feuchtwangen, 1460.

18. - Geburt des Hl. Sebald, Schwäbisch-Gmünd, 1520, Germanisches Nationalmuseum Nürnberg (Gm 191); Meister des Pfullendorfer Altars, Mariengeburt, um 1500, Staatsgalerie Stuttgart (Inv. 1703, aus der Hohenzollernsammlung Sigmaringen); Meister der Lichtenthaler Marienflügel der Zisterzienserinnenkirche Lichtenthal bei Baden-Baden, 1489, Staatliche Kunsthalle (Inv. 806a und b) oder aus der gleichen Abtei die - verlorene - Darstellung der drei Jungfrauen mit dem Hl. Nikolaus, s. http://www.inschriften.net/baden-baden-und-landkreis-rastatt/inschrift/nr/ di078-0137.html, Aufruf 17.11.2018; sowie die Tapisserie zum Leben der Hl. Odile, um 1420, Strasbourg, Musée de l'œuvre de Notre-Dame (D. 22.9820.2). Außerdem aus dem Bereich der Buchillustrationen vgl. etwa jene im Epos der «Sieben weisen Meister», rheinfränkisch, 1471 mit kolorierten Federzeichnungen des Frankfurter Goldschmieds Hans Dirmstein, Universitätsbibliothek Frankfurt (Ms.germ.qu.12), s. vor allem f. 50r.

19. - MINUTOLI, Julius von: Das kaiserliche Buch Markgrafen Albrecht Achilles. Kurfürstliche Periode 1470-1486. 1850/51, Nachdruck Osnabrück 1984, S. 520, dort auch zum in diesem Artikel später beschriebenen Bett der Plassenburg.

20. - Sog. Bett der Maria von Preußen, ebenfalls einer Hohenzollernprinzessin, die Markgraf Christian 1604 geheiratet und auf der Plassenburg das Beilager abgehalten hatte. Sie habe das Bett der Plassenburg gestiftet, s. BACHMANN/Erich, SEELIG, Lorenz/STIERHOF, Horst H.: Amtlicher Führer Plassenburg ob Kulmbach. München 1996, S. 48. Dauerleihgabe des Bayerischen Nationalmuseums, Nadelholz, farbig gefasst, vergoldet. Verkauf der Textilien ab 1728, s. ebda.

21. - Bayerisches Nationalmuseum, München, Tirol (?) um 1500/19. Jh., Zirbenholz, Tanne, Fichte, Schnitzwerk Linde (MA 286) und Bett um 1500, Zirbenholz (MA 2876).

22. - Kissenhülle mit Schlaufenverschluss, 1596, Germanisches Nationalmuseum Nürnberg (Gew 2595-2600), Gedächtnisdecke für Hieronymus Imhoff, 1571 (Gew 2491), s. auch ZANDER-SEIDEL 1990 S. 344, 360-361.

23. - s. dazu auch KOHWAGNER-NIKOLAI, Tanja: "'betruebt und trawrig bisz an mein end". Textiles Totengedächtnis für Hieronymus Imhoff in der zweiten Hälfte des 16. Jahrhunderts». In: Anzeiger des Germanischen Nationalmuseums 2016, S. 205-228.

24. - Auf Grundlage ausführlicher Forschungen schienen mindestens sechs Fassungsvarianten plausibel für die gegebene Zeit und Ort, für keine Version sprach so viel, dass sie einer Vollrekonstruktion hätte Vorschub leisten dürfen. S. dazu auch den Beitrag von PELLUDAT, Inga/ KARNATZ, Sebastian/PIERETH, Uta: «Alles wie neu? Vom musealen Umgang mit Leerstellen bei der Objektrekonstruktion. "In: Museen - Orte des Authentischen? Hg. Römisch-Germanisches Zentralmuseum/Leibniz-Gesellschaft. Mainz 2019.

25. - Die gründlichen Recherchen dazu führte im Vorfeld Dr. Tanja Kohwagner-Nikolai, München aus. Das Bett selbst wurde von den Schreiner- und Tapeziererwerkstätten der Bayerischen Schlösserverwaltung umgesetzt.

26. - s. dazu auch ALBRECHT, Thorsten : Schrank Butze Bett. Vom Mittelalter bis ins 20. Jahrhundert am Beispiel der Lüneburger Heide. Petersberg 2001, und EDER MATT, Katharina: Wie sie sich betten. Basel 1994.

27. - Außer großen oder kleinen Betten wird außerdem ein «ludenn Bett» erwähnt, ein Tagesbett, vorzugsweise mit ledernen oder gewirkten Überdecken. Dass nur ein «Strohsack» des Torwächters und ein weiterer gestreifter genannt werden, heißt gewiss nicht, dass es sonst keine mehr gab. 
28. - S. dazu die oben beschriebenen Quellen und Sach- sowie Liegeüberlieferung bis in die Frühneuzeit hinein, die nur marginale Unterschiede aufweisen und daher Rückschlüsse auf spätmittelalterliche Verhältnisse erlauben.

29. - Im süddeutschen Raum waren Hüllen mit Karos oder Streifen, bezeichnet als «kölnische ziechen» stark verbreitet; feinstes weißes Leinen war dagegen beim Adel üblich für Bezüge und Betttücher.

30. - Vgl. zum Beispiel schon im 14. Jahrhundert Markgräfin Elisabeth, die ihre dementsprechend edle Decke an die Tochter vererbte, s. SEYBOTH, Reinhard: «Burggräfin Elisabeth von Nürnberg in Creußen (1357-ca.1377)». In: Jahrbuch für fränkische Landesforschung 64 (2004), S. 21-38, hier S. 33.

\section{RÉSUMÉS}

Ausgehend von der Aufgabe, die 1945 stark zerstörte Cadolzburg als Herrschaftsmittelpunkt der wichtigen Dynastie der Hohenzollern im 15. Jahrhundert museal zugänglich zu machen und wieder ins öffentliche Bewusstsein $\mathrm{zu}$ heben, nahm die Einrichtung von den zahlreichen schriftlichen Quellen ihren Ausgang. Zur Frage, wie die Schlafkammern aussahen, vermögen besonders zwei Inventare des Jahres 1471 Anhaltspunkte zu vermitteln über die Einrichtung fürstlicher oder gar königlicher deutscher Schlafstätten. Darin gewinnt man über die Anzahl und Abstufung der Bettstätten sowie ihre Ausstattung genauere Vorstellungen, die sich durch Bildquellen der Zeit und nachfolgende Inventare und erhaltene fränkische Hohenzollern-Betten um 1600 ergänzen lassen. Die aus diesen verschiedenen Quellen destillierten Informationen flossen im 2017 eröffneten Museum „HerrschaftsZeiten! Erlebnis Cadolzburg“ in Präsentationsformen ein, die den Besuchern Vorstellungshilfen über die damalige Raumausstattung und v.a. auch über verschiedene höfische Schlafstätten geben. Dabei wurde auf die Anmutung der einst hochwertigen textilen Ausstattung eines Fürstenbettes weitgehend verzichtet, jedoch der Liegekomfort auf verschiedenen Unterbetten, Kissen und Keilen nachvollziehbar gestaltet.

\section{INDEX}

Schlüsselwörter : Franconie, Hohenzollern, Bavière, Cadolzburg, 15. Jahrhundert, Archiven, Museographie, Bettwäsche, Schlafzimmer

\section{AUTEUR}

\section{UTA PIERETH}

Bayerische Schlösserverwaltung, München, Museumsreferentin Cadolzburg

Uta.Piereth@bsv.bayern.de 